\title{
BIDUALITY AND DENSITY IN LIPSCHITZ FUNCTION SPACES
}

\author{
A. JIMÉNEZ-VARGAS, J.M. SEPULCRE, AND MOISÉS VILLEGAS-VALLECILLOS
}

\begin{abstract}
For pointed compact metric spaces $(X, d)$, we address the biduality problem as to when the space of Lipschitz functions $\operatorname{Lip}_{0}(X, d)$ is isometrically isomorphic to the bidual of the space of little Lipschitz function $\operatorname{lip}_{0}(X, d)$, and show that this is the case whenever the closed unit ball of $\operatorname{lip}_{0}(X, d)$ is dense in the closed unit ball of $\operatorname{Lip}_{0}(X, d)$ with respect to the topology of pointwise convergence. Then we apply our density criterion to prove in an alternate way the real version of a classical result which asserts that $\operatorname{Lip}_{0}\left(X, d^{\alpha}\right)$ is isometrically isomorphic to $\operatorname{lip}_{0}\left(X, d^{\alpha}\right)^{* *}$ for any $\alpha \in(0,1)$.
\end{abstract}

\section{INTRODUCTION}

Let $(X, d)$ be a pointed compact metric space with a base point denoted by 0 and let $\mathbb{K}$ be the field of real or complex numbers. The Lipschitz space $\operatorname{Lip}_{0}(X, d)$ is the Banach space of all Lipschitz functions $f: X \rightarrow \mathbb{K}$ for which $f(0)=0$, endowed with the Lipschitz norm

$$
\operatorname{Lip}_{d}(f)=\sup \left\{\frac{|f(x)-f(y)|}{d(x, y)}: x, y \in X, x \neq y\right\} .
$$

A Lipschitz function $f: X \rightarrow \mathbb{K}$ satisfying the local flatness condition:

$$
\lim _{t \rightarrow 0} \sup _{0<d(x, y)<t} \frac{\|f(x)-f(y)\|}{d(x, y)}=0
$$

is called a little Lipschitz function, and the little Lipschitz $\operatorname{space} \operatorname{lip}_{0}(X, d)$ is the closed subspace of $\operatorname{Lip}_{0}(X, d)$ formed by all little Lipschitz functions. Furthermore, $\operatorname{Lip}_{0}^{\mathbb{R}}(X, d)$ and $\operatorname{lip}_{0}^{\mathbb{R}}(X, d)$ are the real subspaces of all real-valued functions in $\operatorname{Lip}_{0}(X, d)$ and $\operatorname{lip}_{0}(X, d)$, respectively. These spaces have been largely investigated along the time. See the Weaver's book [10 for references and a complete study.

The biduality problem as to when $\operatorname{Lip}_{0}(X, d)$ is isometrically isomorphic to $\operatorname{lip}_{0}(X, d)^{* *}$ has an interesting history (see [10, p. 99, Notes 3.3] and also [8, 6. Duality]). In this note, we address this question in a similar way as Bierstedt and Summers 2 do for studying the biduals of weighted Banach spaces of analytic functions, and we prove that $\operatorname{Lip}_{0}(X, d)$ is isometrically isomorphic to $\operatorname{lip}_{0}(X, d)^{* *}$ if and only if the closed unit ball of $\operatorname{lip}_{0}(X, d)$ is dense in the closed unit ball of $\operatorname{Lip}_{0}(X, d)$ with respect to the topology of pointwise convergence $\tau_{p}$. This density condition is equivalent to requiring that for each $f \in \operatorname{Lip}_{0}(X, d)$ with $\operatorname{Lip}_{d}(f) \leq 1$, there exists a sequence $\left\{f_{n}\right\}$ in $\operatorname{lip}_{0}(X, d)$ with $\operatorname{Lip}_{d}\left(f_{n}\right) \leq 1$ for all $n \in \mathbb{N}$ such that $\left\{f_{n}(x)\right\}$ converges to $f(x)$ as $n \rightarrow \infty$ for every $x \in X$. Then we apply our density criterion to prove in an alternate way the real version of a classical Johnson's result 7] (see also [1, 5, 10]) which asserts that $\operatorname{Lip}_{0}\left(X, d^{\alpha}\right)$ is isometrically isomorphic to $\operatorname{lip}_{0}\left(X, d^{\alpha}\right)^{* *}$ for any $\alpha \in(0,1)$.

Date: September 7, 2018.

2010 Mathematics Subject Classification. 46E10, 46E15, 46J10.

Key words and phrases. Lipschitz function, little Lipschitz function, Hölder function, Lipschitz-free Banach space.

This research was partially supported by MICINN under project MTM 2010-17687. 


\section{THE RESUlTs}

Johnson 7 proved that the closed linear subspace of $\operatorname{Lip}_{0}(X, d)^{*}$ spanned by the evaluation functionals $\delta_{x}: \operatorname{Lip}_{0}(X, d) \rightarrow \mathbb{K}$, given by $\delta_{x}(f)=f(x)$ with $x \in X$, is a predual of $\operatorname{Lip}_{0}(X, d)$. The terminology Lipschitz-free Banach space of $X$ and the notation $\mathcal{F}(X)$ for this predual of $\operatorname{Lip}_{0}(X, d)$ are due to Godefroy and Kalton [6]. Namely, the evaluation map $Q_{X}: \operatorname{Lip}_{0}(X, d) \rightarrow \mathcal{F}(X)^{*}$ defined by

$$
Q_{X}(f)(\gamma)=\gamma(f) \quad\left(f \in \operatorname{Lip}_{0}(X, d), \gamma \in \mathcal{F}(X)\right)
$$

is the natural isometric isomorphism. As usual, $B_{E}$ will denote the closed unit ball of a Banach space $E$.

Theorem 1. Let $(X, d)$ be a pointed compact metric space.

(i) The restriction map $R_{X}: \mathcal{F}(X) \rightarrow \operatorname{lip}_{0}(X, d)^{*}$ defined by

$$
R_{X}(\gamma)(f)=\gamma(f) \quad\left(f \in \operatorname{lip}_{0}(X, d), \gamma \in \mathcal{F}(X)\right),
$$

is a nonexpansive linear surjective map.

(ii) $R_{X}$ is an isometric isomorphism from $\mathcal{F}(X)$ onto $\operatorname{lip}_{0}(X, d)^{*}$ if and only if $B_{\operatorname{lip}_{0}(X, d)}$ is dense in $B_{\operatorname{Lip}_{0}(X, d)}$ with respect to the topology of pointwise convergence.

Proof. (i) Since $\mathcal{F}(X) \subset \operatorname{Lip}_{0}(X, d)^{*}$, it is clear that $R_{X}$ is a linear map from $\mathcal{F}(X)$ into $\operatorname{lip}_{0}(X, d)^{*}$ satisfying $\left\|R_{X}(\gamma)\right\| \leq\|\gamma\|$ for all $\gamma \in \mathcal{F}(X)$. We next prove that $R_{X}$ is surjective. To this end, let us recall that De Leeuw's map $\Phi: \operatorname{Lip}_{0}(X, d) \rightarrow C_{b}(\widetilde{X})$ given by

$$
\Phi(f)(x, y)=\frac{f(x)-f(y)}{d(x, y)} \quad\left(f \in \operatorname{Lip}_{0}(X, d),(x, y) \in \widetilde{X}\right)
$$

where $\tilde{X}=\left\{(x, y) \in X^{2}: x \neq y\right\}$, is a linear isometry of $\operatorname{Lip}_{0}(X, d)$ into $C_{b}(\tilde{X})$, the Banach space of bounded continuous scalar-valued functions on $\widetilde{X}$ with the supremum norm, and the image of $\operatorname{lip}_{0}(X, d)$ is contained in $C_{0}(\widetilde{X})$, the closed subspace of functions which vanish at infinity. See, for example, [10, Theorem 2.1.3 and Proposition 3.1.2].

Take $\gamma \in \operatorname{lip}_{0}(X, d)^{*}$. The functional $T: \Phi\left(\operatorname{lip}_{0}(X, d)\right) \rightarrow \mathbb{K}$, defined by $T(\Phi(f))=\gamma(f)$ for all $f \in \operatorname{lip}_{0}(X, d)$, is linear, continuous and $\|T\|=\|\gamma\|$. By the Hahn-Banach theorem, there exists a continuous linear functional $\widetilde{T}: \mathcal{C}_{0}(\widetilde{X}) \rightarrow \mathbb{K}$ such that $\widetilde{T}(\Phi(f))=T(\Phi(f))$ for all $f \in \operatorname{lip}_{0}(X, d)$ and $\|\widetilde{T} \mid=\| T \|$. Now, by the Riesz representation theorem, there exists a finite and regular Borel measure $\mu$ on $\widetilde{X}$ with total variation $\|\mu\|=\|\widetilde{T}\|$ such that

$$
\widetilde{T}(g)=\int_{\widetilde{X}} g d \mu \quad\left(g \in \mathcal{C}_{0}(\widetilde{X})\right)
$$

and thus

$$
\gamma(f)=\int_{\widetilde{X}} \Phi(f) d \mu \quad\left(f \in \operatorname{lip}_{0}(X, d)\right)
$$

If we now define

$$
\widetilde{\gamma}(f)=\int_{\widetilde{X}} \Phi(f) d \mu \quad\left(f \in \operatorname{Lip}_{0}(X, d)\right)
$$

it is clear that $\widetilde{\gamma} \in \operatorname{Lip}_{0}(X, d)^{*}$ and $\widetilde{\gamma}(f)=\gamma(f)$ for all $f \in \operatorname{lip}_{0}(X, d)$. It remains to show that $\widetilde{\gamma}$ is $\tau_{p}$-continuous on $B_{\operatorname{Lip}_{0}(X, d)}$. Thus, let $\left\{f_{i}\right\}$ be a net in $B_{\operatorname{Lip}_{0}(X, d)}$ which converges pointwise on $X$ to zero. Then $\left\{\Phi\left(f_{i}\right)\right\}$ converges pointwise on $\widetilde{X}$ to zero and, since $\left|\Phi\left(f_{i}\right)(x, y)\right| \leq\left\|\Phi\left(f_{i}\right)\right\|_{\infty}=\operatorname{Lip}_{d}\left(f_{i}\right) \leq 1$ for all $i \in I$ and for all $(x, y) \in \widetilde{X}$, it follows that $\left\{\widetilde{\gamma}\left(f_{i}\right)\right\}$ converges to 0 by the Lebesgue's bounded convergence theorem. This completes the proof of (i). 
(ii) Assume that $B_{\operatorname{lip}_{0}(X, d)}$ is $\tau_{p}$-dense in $B_{\operatorname{Lip}_{0}(X, d)}$. Fix $\gamma \in \mathcal{F}(X)$ and let $f \in B_{\operatorname{Lip}_{0}(X, d)}$. Then there exists a net $\left\{f_{i}\right\}$ in $B_{\operatorname{lip}_{0}(X, d)}$ which converges to $f$ in the topology of pointwise convergence. Since $\gamma$ is $\tau_{p}$-continuous on $B_{\operatorname{Lip}_{0}(X, d)}$ and it is satisfied that

$$
\left|\gamma\left(f_{i}\right)\right|=\left|R_{X}(\gamma)\left(f_{i}\right)\right| \leq\left\|R_{X}(\gamma)\right\| \operatorname{Lip}_{d}\left(f_{i}\right) \leq\left\|R_{X}(\gamma)\right\|
$$

for all $i \in I$, it follows that $|\gamma(f)| \leq\left\|R_{X}(\gamma)\right\|$ and so $\|\gamma\| \leq\left\|R_{X}(\gamma)\right\|$. Now, taking into account (i) we conclude that $R_{X}$ is an isometric isomorphism from $\mathcal{F}(X)$ onto $\operatorname{lip}_{0}(X, d)^{*}$.

Conversely, if $B_{\operatorname{lip}_{0}(X, d)}$ is not $\tau_{p}$-dense in $B_{\operatorname{Lip}_{0}(X, d)}$, by the Hahn-Banach theorem there exist a

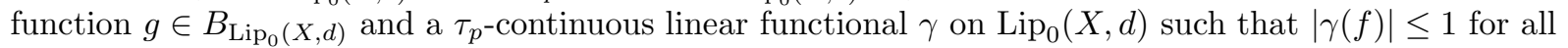
$f \in B_{\operatorname{lip}_{0}(X, d)}$ and $|\gamma(g)|>1$. Since $\gamma \in \mathcal{F}(X)$ and $\left\|R_{X}(\gamma)\right\|=\left\|\left.\gamma\right|_{\operatorname{lip}_{0}(X, d)}\right\| \leq 1<|\gamma(g)| \leq\|\gamma\|$, then $R_{X}$ is not an isometry.

We are now ready to obtain the main result of this note.

Theorem 2. Let $(X, d)$ be a pointed compact metric space. Then the following are equivalent:

(i) $\operatorname{Lip}_{0}(X, d)$ is isometrically isomorphic to $\operatorname{lip}_{0}(X, d)^{* *}$.

(ii) $B_{\operatorname{lip}_{0}(X, d)}$ is dense in $B_{\operatorname{Lip}_{0}(X, d)}$ with respect to the weak* topology.

(iii) $B_{\operatorname{lip}_{0}(X, d)}$ is dense in $B_{\operatorname{Lip}_{0}(X, d)}$ with respect to the topology of pointwise convergence.

(iv) For each $f \in B_{\operatorname{Lip}_{0}(X, d)}$, there exists a sequence $\left\{f_{n}\right\}$ in $B_{\operatorname{lip}_{0}(X, d)}$ such that $\left\{f_{n}(x)\right\}$ converges to $f(x)$ as $n \rightarrow \infty$ for every $x \in X$.

Proof. If (i) holds, then (ii) follows by the Goldstine theorem; but (ii) is the same as (iii) since the weak* topology agrees with the topology of pointwise convergence on bounded subsets of $\operatorname{Lip}_{0}(X, d)$ by 7. Corollary 4.4]. If (iii) is true, then $R_{X}^{*}$ is an isometric isomorphism from $\operatorname{lip}_{0}(X, d)^{* *}$ onto $\mathcal{F}(X)^{*}$ by Theorem 1, hence the composition $Q_{X}^{-1} \circ R_{X}^{*}$ is an isometric isomorphism from $\operatorname{lip}_{0}(X, d)^{* *}$ onto $\operatorname{Lip}_{0}(X, d)$ and so we obtain (i).

In order to prove that (ii) is equivalent to (iv), notice that, by [7, Corollary 4.4], the family of sets

$$
U\left(f_{0} ; n, x_{1}, \ldots, x_{n}, \varepsilon\right):=\left\{f \in B_{\operatorname{Lip}_{0}(X, d)}:\left|f\left(x_{i}\right)-f_{0}\left(x_{i}\right)\right|<\varepsilon, \forall i=1, \ldots, n\right\}
$$

with $f_{0} \in B_{\operatorname{Lip}_{0}(X, d)}, n \in \mathbb{N}, x_{1}, \ldots, x_{n} \in X$ and $\varepsilon>0$, is a basis of the relative weak* topology on $B_{\operatorname{Lip}_{0}(X, d)}$.

Suppose now that (ii) holds and let $f_{0} \in B_{\operatorname{Lip}_{0}(X, d)}$. Given $x \in X$ and $n \in \mathbb{N}$, the set $U\left(f_{0} ; 1, x, 1 / n\right)$ is a weak* neighborhood of $f_{0}$ relative to $B_{\operatorname{Lip}_{0}(X, d)}$. Then, by (ii), for each $n \in \mathbb{N}$ there exists $f_{n} \in B_{\operatorname{lip}_{0}(X, d)}$ such that $f_{n} \in U\left(f_{0} ; 1, x, 1 / n\right)$, that is, $\left|f_{n}(x)-f_{0}(x)\right|<1 / n$. Hence $\left\{f_{n}(x)\right\}$ converges to $f_{0}(x)$ as $n \rightarrow \infty$ and we conclude that (ii) implies (iv). Conversely, assume that (iv) is valid and let $f_{0} \in B_{\operatorname{Lip}_{0}(X, d)}$. Take $U\left(f_{0} ; p, x_{1}, \ldots, x_{p}, \varepsilon\right)$ with $p \in \mathbb{N}, x_{1}, \ldots, x_{p} \in X$ and $\varepsilon>0$. By (iv), there is a sequence $\left\{f_{n}\right\}$ in $B_{\operatorname{lip}_{0}(X, d)}$ such that $\left\{f_{n}(x)\right\}$ converges to $f_{0}(x)$ as $n \rightarrow \infty$ for every $x \in X$. In particular, for each $i \in\{1, \ldots, p\}$, there is a $m_{i} \in \mathbb{N}$ for which $\left|f_{n}\left(x_{i}\right)-f_{0}\left(x_{i}\right)\right|<\varepsilon$ whenever $n \geq m_{i}$. Now, if $m=\max \left\{m_{1}, \ldots, m_{p}\right\}$, then $f_{m} \in U\left(f_{0} ; p, x_{1}, \ldots, x_{p}, \varepsilon\right)$ and (ii) follows.

It is known that $\operatorname{Lip}_{0}(X, d)$ is isometrically isomorphic to $\operatorname{lip}_{0}(X, d)^{* *}$ for a large class of metric spaces $(X, d)$ as, for example, the Hölder spaces $\left(X, d^{\alpha}\right), 0<\alpha<1$ [1, 5, 7].

Remark 3. The proof of Theorem Q shows that if one of its statements holds, then the map $Q_{X}^{-1} \circ R_{X}^{*}$ is an isometric isomorphism from $\operatorname{lip}_{0}(X, d)^{* *}$ onto $\operatorname{Lip}_{0}(X, d)$. For any $\phi \in \operatorname{lip}_{0}(X, d)^{* *}$ and $x \in X$, an 
easy verifications yields

$$
\begin{aligned}
\left(Q_{X}^{-1} \circ R_{X}^{*}\right)(\phi)(x) & =\delta_{x}\left(\left(Q_{X}^{-1} \circ R_{X}^{*}\right)(\phi)\right) \\
& =Q_{X}\left(\left(Q_{X}^{-1} \circ R_{X}^{*}\right)(\phi)\right)\left(\delta_{x}\right) \\
& =Q_{X}\left(Q_{X}^{-1}\left(R_{X}^{*}(\phi)\right)\right)\left(\delta_{x}\right) \\
& =R_{X}^{*}(\phi)\left(\delta_{x}\right) \\
& =\phi\left(R_{X}\left(\delta_{x}\right)\right) \\
& =\phi\left(\delta_{x}\right) .
\end{aligned}
$$

This identification is the same as that obtained by De Leeuw [5], Johnson [7] and Bade, Curtis and Dales [1] between the spaces $\operatorname{Lip}_{0}\left(X, d^{\alpha}\right)$ and $\operatorname{lip}_{0}\left(X, d^{\alpha}\right)^{* *}(0<\alpha<1)$.

The pointwise approximation condition given by the assertion (iv) of Theorem 2 can be verified to recover two classical results about the biduality problem of $\operatorname{Lip}_{0}\left(X, d^{\alpha}\right)(0<\alpha<1)$. The former is due to Ciesielski [4] and the latter to De Leeuw [5].

Example 4. Let $\alpha \in(0,1)$ and let $[0,1]$ be the unit interval with the usual metric d. Then $\operatorname{Lip}_{0}\left([0,1], d^{\alpha}\right)$ is isometrically isomorphic to $\operatorname{lip}_{0}\left([0,1], d^{\alpha}\right)^{* *}$.

Proof. Fix $f \in B_{\operatorname{Lip}_{0}\left([0,1], d^{\alpha}\right]}$ and, for each $n \in \mathbb{N}$, let $B_{n}(f, \cdot)$ denote the $n$th Bernstein polynomial for $f$ defined by

$$
B_{n}(f, x)=\sum_{k=0}^{n} f\left(\frac{k}{n}\right)\left(\begin{array}{l}
n \\
k
\end{array}\right) x^{k}(1-x)^{n-k} \quad(x \in[0,1]) .
$$

Then $B_{n}(f, \cdot)$ also belongs to $B_{\operatorname{Lip}_{0}\left([0,1], d^{\alpha}\right)}$ (see [3] for an elementary proof) while the fact that

$$
\begin{aligned}
\left|B_{n}(f, x)-B_{n}(f, y)\right| & \leq \sum_{k=0}^{n}\left|f\left(\frac{k}{n}\right)\right|\left(\begin{array}{l}
n \\
k
\end{array}\right)\left|x^{k}(1-x)^{n-k}-y^{k}(1-y)^{n-k}\right| \\
& \leq|x-y| \sum_{k=0}^{n}\left|f\left(\frac{k}{n}\right)\right|\left(\begin{array}{l}
n \\
k
\end{array}\right) 2 n
\end{aligned}
$$

for all $x, y \in[0,1]$ shows that $B_{n}(f, \cdot) \in B_{\operatorname{lip}_{0}\left([0,1], d^{\alpha}\right)}$. Since $\left\{B_{n}(f, \cdot)\right\}_{n \in \mathbb{N}}$ converge to $f$ uniformly on $[0,1]$, the example is proved by Theorem 2 .

Example 5. Let $0<\alpha<1$ and let $\mathbb{T}$ be the quotient additive group $\mathbb{R} / 2 \pi \mathbb{Z}$ with the distance

$$
d(t+2 \pi \mathbb{Z}, s+2 \pi \mathbb{Z})=\min \{|t-s|,|t-s-2 \pi|,|t-s+2 \pi|\} \quad(t, s \in[0,2 \pi)) .
$$

Then $\operatorname{Lip}_{0}\left(\mathbb{T}, d^{\alpha}\right)$ is isometrically isomorphic to $\operatorname{lip}_{0}\left(\mathbb{T}, d^{\alpha}\right)^{* *}$.

Proof. We apply similar arguments to those of [5, Lemma 2.8] and use some results from harmonic analysis (see [9]). We identify each equivalence class $t+2 \pi \mathbb{Z}$ with the point $t \in[0,2 \pi)$. Let $f \in B_{\operatorname{Lip}_{0}\left(\mathbb{T}, d^{\alpha}\right)}$. For each $n \in \mathbb{N}$, let $K_{n}$ be the Fejér kernel defined by

$$
K_{n}(t)=\sum_{j=-n}^{n}\left(1-\frac{|j|}{n+1}\right) e^{i j t}=\frac{1}{n+1}\left(\frac{\sin \frac{n+1}{2}}{\sin \frac{t}{2}}\right)^{2} \quad(t \in[0,2 \pi)) .
$$

Then the convolution

$$
\left(K_{n} * f\right)(t)=\frac{1}{2 \pi} \int_{0}^{2 \pi} K_{n}(\tau) f(t-\tau) d \tau \quad(t \in[0,2 \pi))
$$


coincides with the Fejér mean

$$
\sigma_{n}(f, t)=\sum_{j=-n}^{n}\left(1-\frac{|j|}{n+1}\right) \widehat{f}(j) e^{i j t} \quad(t \in[0,2 \pi)),
$$

where $\widehat{f}(j)$ is the $j$ th Fourier coefficient of $f$. Given $t, s \in[0,2 \pi)$, we have

$$
\begin{aligned}
\left|\sigma_{n}(f, t)-\sigma_{n}(f, s)\right| & \leq \sum_{j=-n}^{n}\left|1-\frac{|j|}{n+1}\right||\widehat{f}(j)|\left|e^{i j t}-e^{i j s}\right| \\
& \leq \sum_{j=-n}^{n}\left|1-\frac{|j|}{n+1}\right| \frac{\pi^{\alpha}|j|^{1-\alpha}}{2}(4 \pi n)^{n}(e-1) d(t, s)
\end{aligned}
$$

and therefore $\sigma_{n}(f, \cdot) \in \operatorname{lip}_{0}\left(\mathbb{T}, d^{\alpha}\right)$. Moreover,

$$
\begin{aligned}
\left|\sigma_{n}(f, t)-\sigma_{n}(f, s)\right| & =\left|\left(K_{n} * f\right)(t)-\left(K_{n} * f\right)(s)\right| \\
& \leq \frac{1}{2 \pi} \int_{0}^{2 \pi}\left|K_{n}(\tau)\right||f(t-\tau)-f(s-\tau)| d \tau \\
& \leq \operatorname{Lip}_{d^{\alpha}}(f) d(t, s)^{\alpha} \frac{1}{2 \pi} \int_{0}^{2 \pi} K_{n}(\tau) d \tau \\
& =\operatorname{Lip}_{d^{\alpha}}(f) d(t, s)^{\alpha},
\end{aligned}
$$

and so $\operatorname{Lip}_{d^{\alpha}}\left(\sigma_{n}(f, \cdot)\right) \leq \operatorname{Lip}_{d^{\alpha}}(f) \leq 1$. Now take $\beta_{n}(f, \cdot)=\sigma_{n}(f, \cdot)-\sigma_{n}(f, 0)$ which is in $B_{\operatorname{lip}_{0}\left(\mathbb{T}, d^{\alpha}\right)}$. By the Fejér theorem, $\left\{\sigma_{n}(f, \cdot)\right\}_{n \in \mathbb{N}}$ converges pointwise on $\mathbb{T}$ to $f$, and so does $\left\{\beta_{n}(f, \cdot)\right\}_{n \in \mathbb{N}}$. Then the desired conclusion follows from Theorem 2

Our density criterion serves to give another proof of the real version of an important result by Johnson [7. Theorem 4.7] and Bade, Curtis and Dales [1, Theorem 3.5].

Corollary 6. Let $(X, d)$ be a pointed compact metric space and let $\alpha \in(0,1)$. Then $\operatorname{Lip}_{0}^{\mathbb{R}}\left(X, d^{\alpha}\right)$ is isometrically isomorphic to $\operatorname{lip}_{0}^{\mathbb{R}}\left(X, d^{\alpha}\right)^{* *}$.

Proof. Let $f \in B_{\operatorname{Lip}_{0}^{\mathbb{R}}\left(X, d^{\alpha}\right)}$. We claim that for each $n \in \mathbb{N}$ and each finite set $F \subset X$, there exists a function $h \in \operatorname{lip}^{\mathbb{R}}\left(X, d^{\alpha}\right)$ such that $\operatorname{Lip}_{d^{\alpha}}(h) \leq 1+1 / n$ and $h(x)=f(x)$ for all $x \in F$. The notation $\operatorname{lip}^{\mathbb{R}}\left(X, d^{\alpha}\right)$ and later $\operatorname{Lip}^{\mathbb{R}}\left(X, d^{\gamma}\right)$ might be self-explanatory.

Consider $F=\left\{x_{1}, \ldots, x_{m}\right\}$ for some $m \in \mathbb{N}$ and there is no loss of generality in assuming that $f\left(x_{m}\right) \leq f\left(x_{m-1}\right) \leq \ldots \leq f\left(x_{1}\right)$. If $m=1$, we set $h(x)=f\left(x_{1}\right)$ for all $x \in X$. Now let $m \geq 2$ and we also may assume $f \geq 0$, for otherwise we can replace $f$ by $f+\|f\|_{\infty}$. Let

$$
\begin{aligned}
& \gamma=\min \left(\left\{\alpha+\frac{e \ln \left(1+\frac{1}{n}\right)}{\operatorname{diam}(X)} d\left(x_{j}, x_{k}\right): j, k \in\{1, \ldots, m\}, j \neq k\right\} \cup\{1\}\right), \\
& \rho=\max \left\{\frac{\left|f\left(x_{k}\right)-f\left(x_{j}\right)\right|}{d\left(x_{k}, x_{j}\right)^{\gamma}}: j, k \in\{1, \ldots, m\}, j \neq k\right\} .
\end{aligned}
$$

For each $j \in\{1, \ldots, m\}$, define $g_{j}: X \rightarrow \mathbb{R}$ by

$$
g_{j}(x)=\max \left\{f\left(x_{j}\right)-\rho d\left(x_{j}, x\right)^{\gamma}, 0\right\} .
$$

Notice that $0<\alpha<\gamma \leq 1$ and therefore $g_{j} \in \operatorname{Lip}^{\mathbb{R}}\left(X, d^{\gamma}\right) \subset \operatorname{lip}^{\mathbb{R}}\left(X, d^{\alpha}\right)$ with

$$
\operatorname{Lip}_{d^{\alpha}}\left(g_{j}\right) \leq \operatorname{Lip}_{\gamma}\left(g_{j}\right) \operatorname{diam}(X)^{\gamma-\alpha} \leq \rho \operatorname{diam}(X)^{\gamma-\alpha} .
$$


We now check that the function $h=\max \left\{g_{1}, \ldots, g_{m}\right\}$ satisfies the required conditions. It is known that $h$ is in $\operatorname{lip}^{\mathbb{R}}\left(X, d^{\alpha}\right)$ and $\operatorname{Lip}_{d^{\alpha}}(h) \leq \max \left\{\operatorname{Lip}_{d^{\alpha}}\left(g_{1}\right), \ldots, \operatorname{Lip}_{d^{\alpha}}\left(g_{m}\right)\right\}$. Now, given $j \in\{1, \ldots, m\}$, for some $k, i \in\{1, \ldots, m\}$ with $k \neq i$, we have

$$
\begin{aligned}
\operatorname{Lip}_{d^{\alpha}}\left(g_{j}\right) & \leq \rho \operatorname{diam}(X)^{\gamma-\alpha} \\
& =\frac{\left|f\left(x_{k}\right)-f\left(x_{i}\right)\right|}{d\left(x_{k}, x_{i}\right)^{\gamma}} \operatorname{diam}(X)^{\gamma-\alpha} \\
& \leq \operatorname{Lip}_{d^{\alpha}}(f)\left(\frac{\operatorname{diam}(X)}{d\left(x_{k}, x_{i}\right)}\right)^{\gamma-\alpha} \\
& \leq\left(\frac{\operatorname{diam}(X)}{d\left(x_{k}, x_{i}\right)}\right)^{\frac{e \ln \left(1+\frac{1}{n}\right)}{\operatorname{diam}(X)} d\left(x_{k}, x_{i}\right)} \\
& \leq 1+\frac{1}{n}
\end{aligned}
$$

The last inequality follows from the fact that the function $t \mapsto(t / \operatorname{diam}(X))^{t e \ln (1+1 / n) / \operatorname{diam}(X)}$ for all $t>0$ has a minimum value of $1 /(1+1 / n)$. Hence $\operatorname{Lip}_{d^{\alpha}}(h) \leq 1+1 / n$ as required. Now let $j, k \in\{1, \ldots, m\}$. If $j \leq k$, it is immediate that $g_{k}\left(x_{j}\right) \leq f\left(x_{k}\right) \leq f\left(x_{j}\right)=g_{j}\left(x_{j}\right)$, whereas that if $k<j$, we have $\left|f\left(x_{k}\right)-f\left(x_{j}\right)\right| / d\left(x_{k}, x_{j}\right)^{\gamma} \leq \rho$, hence $f\left(x_{k}\right)-\rho d\left(x_{k}, x_{j}\right)^{\gamma} \leq f\left(x_{j}\right)$ and thus $g_{k}\left(x_{j}\right) \leq g_{j}\left(x_{j}\right)$. Therefore $h\left(x_{j}\right)=g_{j}\left(x_{j}\right)=f\left(x_{j}\right)$ for all $j \in\{1, \ldots, m\}$. The claim follows.

Now fix $n \in \mathbb{N}$ and, for each $x \in X$, let $B(x, 1 / n)=\left\{y \in X: d(y, x)^{\alpha}<1 / n\right\}$. By the compactness of $X$, there is a finite subset $F_{n}$ of $X$ such that $X=\bigcup_{x \in F_{n}} B(x, 1 / n)$. We can suppose that the base point $0 \in X$ is in $F_{n}$, for otherwise take the finite set $F_{n} \cup\{0\}$. By the claim, there exists a function $h_{n} \in \operatorname{lip}^{\mathbb{R}}\left(X, d^{\alpha}\right)$ such that $\operatorname{Lip}_{d^{\alpha}}\left(h_{n}\right) \leq 1+1 / n$ and $h_{n}(x)=f(x)$ for all $x \in F_{n}$. Hence $h_{n} \in \operatorname{lip} \mathbb{R}_{0}^{\mathbb{R}}\left(X, d^{\alpha}\right)$. To prove that the sequence $\left\{h_{n}\right\}$ converges pointwise on $X$ to $f$, let $x \in X$. For each $n \in \mathbb{N}$, choose $y_{n} \in F_{n}$ such that $d\left(x, y_{n}\right)^{\alpha}<1 / n$. Note that $h_{n}\left(y_{n}\right)=f\left(y_{n}\right)$ and thus

$$
\begin{aligned}
\left|f(x)-h_{n}(x)\right| & \leq\left|f(x)-f\left(y_{n}\right)\right|+\left|f\left(y_{n}\right)-h_{n}(x)\right| \\
& \leq\left|f(x)-f\left(y_{n}\right)\right|+\left|h_{n}\left(y_{n}\right)-h_{n}(x)\right| \\
& \leq\left(\operatorname{Lip}_{d^{\alpha}}(f)+\operatorname{Lip}_{d^{\alpha}}\left(h_{n}\right)\right) d\left(x, y_{n}\right)^{\alpha} \\
& \leq\left(2+\frac{1}{n}\right) \frac{1}{n} .
\end{aligned}
$$

Hence the sequence $\left\{h_{n}(x)\right\}$ converges to $f(x)$ as $n \rightarrow \infty$. Finally, let $r_{n}=\max \left\{1, \operatorname{Lip}_{d^{\alpha}}\left(h_{n}\right)\right\}$ and $f_{n}=h_{n} / r_{n}$ for each $n \in \mathbb{N}$. It is clear that $\left\{f_{n}\right\}$ is a sequence in $B_{\operatorname{lip}_{0}^{\mathbb{R}}\left(X, d^{\alpha}\right)}$ that converges pointwise to $f$ on $X$. Then the corollary follows from Theorem 2

\section{REFERENCES}

1. W. G. Bade, P. C. Curtis, Jr. and H. G. Dales, Amenability and weak amenability for Beurling and Lipschitz algebras, Proc. London Math. Soc. (3) 55 (1987), 359-377.

2. K. D. Bierstedt and W. H. Summers, Biduals of weighted Banach spaces of analytic functions, J. Austral. Math. Soc. Ser. A 54 (1993), no. 1, 70-79.

3. B. M. Brown, D. Elliott and D. F. Paget, Lipschitz constants for the Bernstein polynomials of a Lipschitz continuous function, J. Approx. Theory 49 (1987), no. 2, 196-199.

4. Z. Ciesielski, On the isomorphisms of the spaces $H_{\alpha}$ and $m$, Bull. Acad. Polon. Sci. 8 (1960), 217-222.

5. K. de Leeuw, Banach spaces of Lipschitz functions, Studia Math. 21 (1961), 55-66.

6. G. Godefroy and N. J. Kalton, Lipschitz-free Banach spaces, Studia Math. 159 (2003), 121-141.

7. J. A. Johnson, Banach spaces of Lipschitz functions and vector-valued Lipschitz functions, Trans. Amer. Math. Soc. 148 (1970), 147-169.

8. N. J. Kalton, Spaces of Lipschitz and Hölder functions and their applications, Collect. Math. 55 (2004), 171-217. 
9. Y. Katznelson, An introduction to harmonic analysis, Third edition, Cambridge Mathematical Library, Cambridge University Press, Cambridge, 2004.

10. N. Weaver, Lipschitz Algebras, World Scientific Publishing Co., Singapore, 1999.

Departamento de Matemáticas, Universidad de Almería, 04120 Almería, Spain

E-mail address: ajimenez@ual.es

Departamento de Análisis Matemático, Universidad de Alicante, 03080 Alicante, Spain

E-mail address: JM.Sepulcre@ua.es

Campus Universitario de Puerto Real, Facultad de Ciencias, 11510 Puerto Real, Cádiz, Spain

E-mail address: moises.villegas@uca.es 